\title{
Rapid dissipation in a current sheet driven by footpoints motions
}

\author{
G. Fruit ${ }^{\star}$ and I. J. D. Craig
}

The University of Waikato, New Zealand

e-mail: fruit@cesr.fr

Received 20 April 2006 / Accepted 11 July 2006

\begin{abstract}
Context. Wave energy dissipation by viscous and resistive damping in magnetic coronal plasmas is addressed. A two and one half dimensional, line-tied magnetic channel is considered whose footpoints are disturbed by buffeting motions of the photosphere.

Aims. The aim of the analysis is to determine how effectively shear Alfvén waves, driven by footpoint motions and resonating in the channel, can be damped by visco-resistive friction.

Methods. The problem is analyzed using a mixture of numerical and analytic techniques. Dynamic simulations, based on a cyclic footpoint driver, show that phase mixing resulting from variations in the magnetic field of the channel is instrumental in setting up a permanent "resonance regime" for the system. Analytic methods are used to analyze the resonance regime and to develop scaling laws for the rate of energy dissipation.

Results. We show that in the general case where the Alfvén velocity profile may be linearized around the resonance, the dissipation rate is "fast" in that it is not limited by small damping coefficients. Although large energies can accumulate within the channel in the limit of very small damping, we show that by tuning the driver to low frequencies, both the energy level and the dissipation rate can be made insensitive to the level of the damping.
\end{abstract}

Key words. magnetohydrodynamics (MHD) - Sun: corona - Sun: magnetic fields - waves

\section{Introduction}

The problem of heating the solar corona remains a major challenge in space physics. Although early studies favoured heating by acoustic waves (Athay \& White 1978), it now seems likely that coronal magnetic field is intrinsically linked to the heating mechanism. Magnetic heating could occur via several distinct processes. One possibility is provided by the visco-resistive damping of Alfvén waves generated in the lower solar atmosphere (Berghmans et al. 1996; Ruderman et al. 1997). An alternate mechanism is provided by Parker's coronal heating hypothesis where heating occurs by in situ Ohmic dissipation at specialized sites, defined by "micro-sheets" in the corona (see also Mikic et al. 1989). In the case of the solar flare, it is the liberation of energy by magnetic reconnection that is expected to account for the explosive release (Priest \& Forbes 2000).

In this study we focus on the visco-resistive dissipation of shear Alfvénic disturbances in generic magnetic configurations characterized by a well-defined Alfvén profile. Although our final aim is to study the visco-resistive damping of Alfvén waves in $X$-point geometries, we restrict our present analysis to a stratified 1D magnetic channel, as in Craig \& Fruit (2005). In fact, as confirmed by the $X$-point study of Fruit \& Craig (2006), wave energy decay in a line-tied one-dimensional field contains much of the essential behaviour in the more complicated 2D problem. Specifically, Craig \& Fruit (2005) studied energy dissipation within a 1D current sheet by considering an initial value problem in which wave energy, present in the system at $t=0$, is resistively and viscously damped. The initial stage of the damping was shown to follow an energy decay law of the form $\exp \left(-\alpha t^{3}\right)$, identical to the one derived by Heyvaerts \& Priest (1983) and

\footnotetext{
* Now at CESR, Toulouse, France
}

subsequent authors. But a radical change of behaviour occurs in the later decay due to the emergence of self-similar modes and/or quasi-eigenmodes, which effectively provide a brake on the rate of energy dissipation.

Our present purpose is to extend the analysis of Craig \& Fruit (2005) by considering a driven problem in which energy is continuously supplied by boundary motions. As motivation we note that since coronal magnetic field lines are effectively "frozen in" to the photosphere, their footpoints are constantly buffeted due to random photospheric motions. If these displacements are fast enough, they generate MHD waves that propagate along the field lines (Hollweg 1987). Inhomogeneities in the magnetic plasma then promote a transfer of wave energy into heat via phase mixing (Heyvaerts \& Priest 1983) and/or resonant absorption (Ionson 1978; Tirry et al. 1997; Fruit et al. 2002).

At present, it is not clear whether resistive or viscous damping accounts for the bulk of wave energy losses. The main difficulty with resistive damping (see e.g. Priest \& Forbes 2000) is the low resistivity of typical coronal plasmas. Since the dimensionless resistivity $\eta$ is an inverse Lundquist number of order $10^{-14}$ (Spitzer 1962), rapid Ohmic dissipation cannot be achieved in the absence of huge, possibly non-physical, current densities. It seems that viscous damping should be more robust since viscosity is not burdened by such weak damping coefficients $(v \gg \eta)$. Indeed Hollweg (1986) has already emphasized theoretically that viscosity should be important in a wide variety of coronal processes. Yet the difficulty remains, that viscous dissipation is highly anisotropic in a magnetically stratified plasma, and that an accurate description requires a detailed tensor description (Braginskii 1965). It appears therefore, that viscous damping could depend sensitively on the magnetic geometry of the plasma. 
In the present study we take the simplest approach and assume collision Laplacian forms for the viscous and resistive terms. Although anisotropic viscous effects are neglected, this assumption will be least damaging if the damping mechanism is found to be insensitive to the amplitude of the dissipation coefficients. In fact we will show that resonant effects deriving from oscillatory footpoint motions can lead to damping rates which are effectively independent of the dissipation coefficients. This result, first pointed out by Heyvaerts \& Priest (1983), is true as long as the Alfvén speed can be linearized around the resonant line. In what follows we consider more general forms for the Alfvén profile and by introducing the quality factor $Q$, discuss not only the dissipation rate, but also the energy stored in the resonating system.

In Sect. 2 we describe the MHD formulation of the problem. We point out that resonance effects lead to a blow-up of the ideal system and, in the case of finite dissipation, we employ numerical results to highlight the main features of the damping. These results are interpreted analytically in Sect. 3 where theoretical scaling laws are derived to explain the bulk energetics. In particular, we point out in Sect. 3.6 that when the system is driven appropriately, both the energy dissipation and the energy stored in the resonator become insensitive to the damping coefficients.

\section{Shear Alfvén waves in a current sheet}

\subsection{Introduction}

We adopt the two and a half dimensional planar MHD equations as described in Craig \& Fruit (2005). The basic geometry is that of a macroscopic current channel of length $2 L$ sandwiched by rigid walls at $y= \pm 1$ (in suitable units). The unperturbed magnetic field $B_{0}(y)$ is assumed to vanish at the origin but can take any smooth variation along $y$. This simple geometry, shown in Fig. 1, can be regarded as a 1D prototype for more complex $X$-point configurations.

The Alfvén velocity is given by

$v_{\mathrm{A}}(y)=\frac{B_{0}(y)}{\sqrt{\mu_{0} \rho(y)}}$

where the density profile $\rho(y)$ is chosen so that the equilibrium magnetic forces are balanced by the pressure gradient. It is the non-uniformity of $v_{\mathrm{A}}(y)$ which promotes phase mixing and leads to rapid dissipation of shear wave disturbances. In particular, footpoint motions drive a set of standing shear Alfvén waves along the $x$-axis which feed energy into the system. Our aim is to determine how this wave energy is damped and to examine how the gradient in the Alfvén speed influences the dissipation rate.

In what follows we adopt a dimensionless formulation in which magnetic field intensities, plasma densities and lengths are scaled according to typical reference values. Velocities and time are then measured in units of the Alfvén speed $v_{\mathrm{A}}$ and the Alfvén time $\tau_{\mathrm{A}}$ respectively. For magnetic plasmas in the low solar atmosphere (low corona, upper chromosphere) the Alfvén time is typically around $10 \mathrm{~s}$.

\subsection{MHD equations and boundary conditions}

It is easily shown that the axial wave fields, $v(x, y, t)$ and $b(x, y, t)$, satisfy the visco-resistive MHD equations

$$
\frac{\partial v}{\partial t}=v_{\mathrm{A}}(y) \frac{\partial b}{\partial x}+v \nabla^{2} v
$$

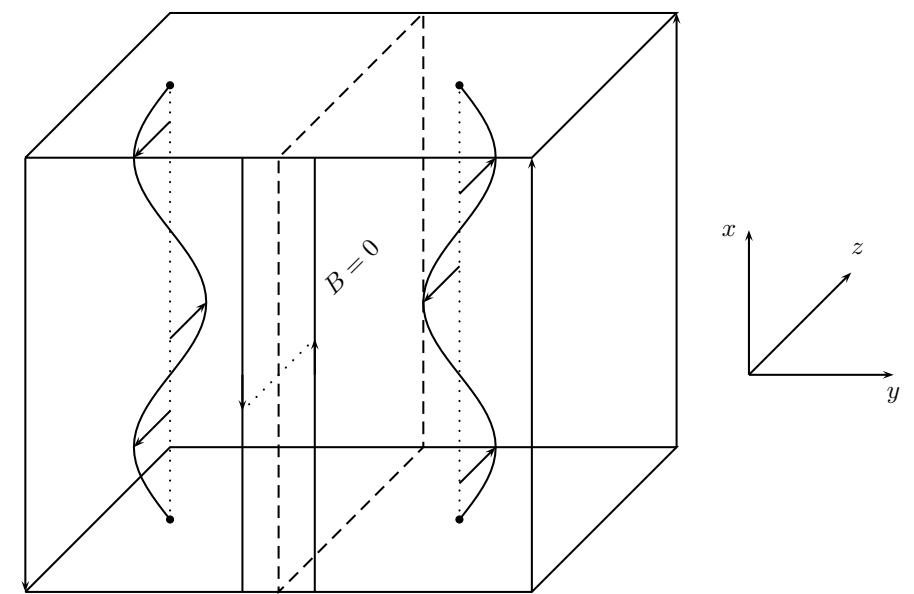

Fig. 1. The geometry of the background magnetic field: $\boldsymbol{B}_{0}=B_{0}(y) \widehat{\boldsymbol{x}}$ and sketch of the shear disturbances propagating along the magnetic field lines.

$\frac{\partial b}{\partial t}=v_{\mathrm{A}}(y) \frac{\partial v}{\partial x}+\eta \nabla^{2} b$

Note that collisional Laplacian forms are assumed to account for dissipation due to viscous and resistive damping (with $v$ and $\eta$ constant). In this case the dimensionless resistivity $\eta$ is an inverse Lundquist number which can be as small as $10^{-14}$ for coronal plasmas. In a magnetic plasma the viscosity is a actually a strong function of the magnetic field (Braginskii 1965) which achieves the hydrodynamic value $v_{0} \sim 10^{-5}$ only in a narrow strip about the neutral line $y=0$ where the magnetic field is sufficiently weak. In stronger fields the cross field viscosity is reduced by $\left(\omega_{B} \tau\right)^{2}$ where $\tau$ is the collision time and $\omega_{B}$ the gyrofrequency. In solar environments, the cross field viscosity may be reduced to the level of the resistivity $\eta$. Here, for analytic simplicity and generality, we consider only a small, uniform value for the viscous coefficient $\nu$. We recognize however, that for accurate computations in general $X$-point topologies, the strongfield Braginskii tensor viscosity should be employed. An accurate viscous term should also be weighted with the inverse mass density, since significant density variations may be required to balance the equilibrium magnetic forces, at least in the case of low beta plasma.

To complete the problem the boundary conditions must be specified. As in Craig \& Fruit (2005) and for numerical simplicity, rigid walls at $y= \pm 1$ imply that both $v$ and $b$ vanish on these surfaces. The conditions on $x= \pm L$ are altered however, since energy is now fed into the system by shearing motions on the left and right hand boundaries. This form of "driving" can be incorporated by superposing appropriate volumetric source terms on to the right hand side of (1) and (2).

Consider, for instance, conditions which represent "antisymmetric" driving, namely

$v(x= \pm L, y, t)= \pm V(y, t)$,

$\frac{\partial b}{\partial x}(x= \pm L, y, t)=0$.

The standard reduction (see Heyvaerts \& Priest 1983; Ruderman 1999)

$W=v(x, y, t)-(x / L) V(y, t)$,

$Z=b(x, y, t)$, 
gives

$$
\begin{aligned}
\frac{\partial W}{\partial t} & =v_{\mathrm{A}}(y) \frac{\partial Z}{\partial x}+v\left(\nabla^{2} W+\frac{x}{L} \frac{\partial^{2} V}{\partial y^{2}}\right)-\frac{x}{L} \frac{\partial V}{\partial t}, \\
\frac{\partial Z}{\partial t} & =v_{\mathrm{A}} \frac{\partial W}{\partial x}+\eta \nabla^{2} Z+\frac{v_{\mathrm{A}}}{L} V,
\end{aligned}
$$

with the new boundary conditions

$$
\begin{aligned}
& W(x= \pm L, y, t)=\frac{\partial Z}{\partial x}(x= \pm L, y, t)=0 \\
& \text { and } \quad W(x, y= \pm 1, t)=Z(x, y= \pm 1, t)=0 .
\end{aligned}
$$

Equations (3), (4) form the basis of our analysis. The system coincides with the "undriven" system of numerous authors (Heyvaerts \& Priest 1983; Hood et al. 1997; Craig \& Fruit 2005) when the volumetric source terms are turned off and it is similar to the system of Ruderman et al. (1997) written in cylindrical coordinates. For the remainder of the paper we specialize to the sinusoidal driving term

$V(y, t)=V_{0}(y) \sin \Omega t$

where the amplitude $V_{0}$ is taken to be a smooth function satisfying $V_{0}( \pm 1)=0$.

\subsection{Fourier representation of the solution}

Since the system (3)-(4) is homogeneous in the $x$-direction, the velocity and magnetic fields may be expanded in Fourier series so that the boundary conditions in $x$ are automatically satisfied. Specifically we take

$$
\begin{aligned}
W(x, y, t) & =\sum_{n=1}^{\infty} W_{n}(y, t) \sin \left(\frac{n \pi x}{L}\right) \\
Z(x, y, t) & =\frac{1}{2} Z_{0}(y, t)+\sum_{n=1}^{\infty} Z_{n}(y, t) \cos \left(\frac{n \pi x}{L}\right)
\end{aligned}
$$

and use the representation

$\frac{x}{L}=\sum_{n=1}^{\infty} s_{n} \sin \frac{n \pi x}{L} \quad$ with $\quad s_{n}=(-1)^{n+1} \frac{2}{n \pi}$.

Each mode is now governed by the system:

$$
\begin{aligned}
\frac{\partial W_{n}}{\partial t} & =-n \omega_{\mathrm{A}} Z_{n}+v\left[-\left(\frac{n \pi}{L}\right)^{2} W_{n}+\frac{\partial^{2} W_{n}}{\partial y^{2}}+s_{n} \frac{\partial^{2} V}{\partial y^{2}}\right]-s_{n} \frac{\partial V}{\partial t}, \\
\frac{\partial Z_{n}}{\partial t} & =n \omega_{\mathrm{A}} W_{n}+\eta\left[-\left(\frac{n \pi}{L}\right)^{2} Z_{n}+\frac{\partial^{2} Z_{n}}{\partial y^{2}}\right],
\end{aligned}
$$

where

$\omega_{\mathrm{A}}(y)=\pi \frac{v_{\mathrm{A}}}{L}$.

In Sect. 3 we provide an analytic description of the damping in the permanent regime of the driven system. For the present let we briefly mention the ideal case $(\eta=v=0)$. It is easy to show that each field line behaves like an harmonic oscillator of frequency $\omega_{\mathrm{A}}=\omega_{\mathrm{A}}(y)$. When the system is driven by a sinusoidal excitation of frequency $\Omega$, a resonant phenomenon develops about the field line whose eigenfrequency matches the driving frequency $\omega_{\mathrm{A}}\left(y_{\mathrm{r}}\right)=\Omega$. Without any dissipative processes these resonant oscillations grow unboundedly in time. That is, using standard methods (see Sect. 3.2) we find that for

$\Omega=n \omega_{\mathrm{A}}\left(y_{\mathrm{r}}\right)$ we obtain a resonance line whose amplitude grows linearly in time according to

$$
\begin{aligned}
& v_{n}\left(y_{\mathrm{r}}, t\right)=(-1)^{n} \frac{V_{0}}{n \pi}(\Omega t \cos \Omega t-\sin \Omega t) \\
& b_{n}\left(y_{\mathrm{r}}, t\right)=(-1)^{n} \frac{V_{0}}{n \pi} \Omega t \sin \Omega t .
\end{aligned}
$$

A key point is that the energy supplied from the boundary can accumulate only in a very narrow region around the resonance. This implies that when viscous and/or resistive effects are included, the resonance energy can be dissipated quite efficiently.

\subsection{Numerical examples of the driven system}

We now turn to a numerical simulation of the full system (9)-(10), using a classical second-order difference scheme. For numerical purposes we assume $L=1, v=10^{-4}$ and $\eta=0$ and specialize to a linear Alfvén profile, namely $\omega_{\mathrm{A}}(y)=\pi y$.

Figure 2 illustrates typical velocity profiles. Shown are a series of snapshots of $W(y, t)$ plotted against $y$, based on the driving parameters $\Omega=V_{0}=1$. The evolution may be decomposed into three distinct phases: (1) the development of corrugations due to phase mixing; (2) the emergence of a resonant line located at $y_{\mathrm{r}} \sim 0.3$; and (3) the establishment of a permanent oscillatory regime with constant amplitude.

In the initial phase, gradients in the Alfvén speed lead to the build up of sharp cross field corrugations. These begin to smooth out when visco-resistive damping becomes effective, after a time of order $(v+\eta)^{1 / 3}$. This stage has been extensively studied by numerous authors (Hood et al. 1997; Craig \& Fruit 2005) and will not be pursued here. Of more interest is the new feature which derives from the driving, namely, the appearance of a resonant line located where $\Omega=\omega_{\mathrm{A}}\left(y_{\mathrm{r}}\right)$ or $y_{\mathrm{r}}=1 / \pi$. Contrary to the ideal case, the oscillations around the resonant line are stabilized by the dissipation mechanism - viscous damping in the present case. We find that the final oscillatory amplitude reaches a well defined maximum determined by the level of the damping. This phase is characterized, not by phase mixing, but by a global oscillation at the driving frequency $\Omega$. Thus while phase mixing is instrumental in setting up the conditions required for resonance, it plays no discernable role once the permanent regime is established.

Figure 3 represents the total energy (kinetic plus magnetic) of the oscillations as a function of time for a range of viscosities (from bottom to top $v=10^{-3}-10^{-4}-10^{-5}$ ). Not surprisingly, the smaller the resistivity is, the longer it takes to reach the permanent stage. More specifically, as can be easily estimated from the diagram, we find that both the duration of the transient phase, as well as the total amount of energy stored in the final regime, scale as $v^{-1 / 3}$. The next section is devoted to an analytic explanation of these features.

\section{Analytic description of the driven system}

Our aim is to develop an analytic description of the driven system and to construct robust visco-resistive scaling laws that account for the energy conversion from the driver into the plasma. We also explore the influence of Alfvén speed profile $v_{\mathrm{A}}(y)$ on the development of the permanent regime.

\subsection{Simplified analytic system}

To interpret the numerical results of Sect. 2.4 we use a simplified version of system (9)-(10) which retains only the dominant 

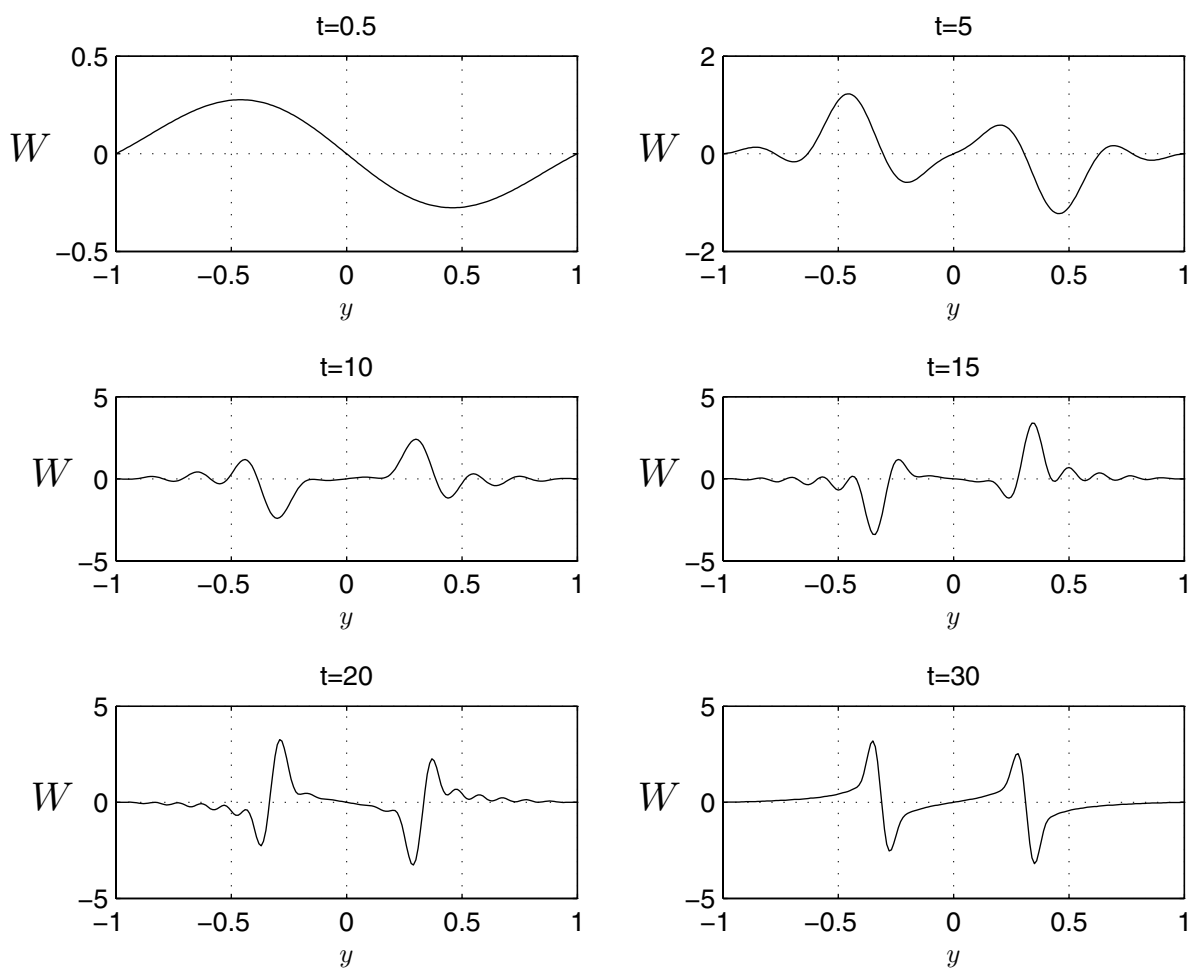

Fig. 2. Temporal sequence of the oscillations during the transient phase. The velocity field $W(y, t)$ is represented as function of $y$ for various times specified above each graph. The resistivity is nought and the viscosity is $v=10^{-4}$. First phase mixing is observed with corrugations across the field lines and then, a resonance effect emerges around a specific field line where most of the energy is concentrated.

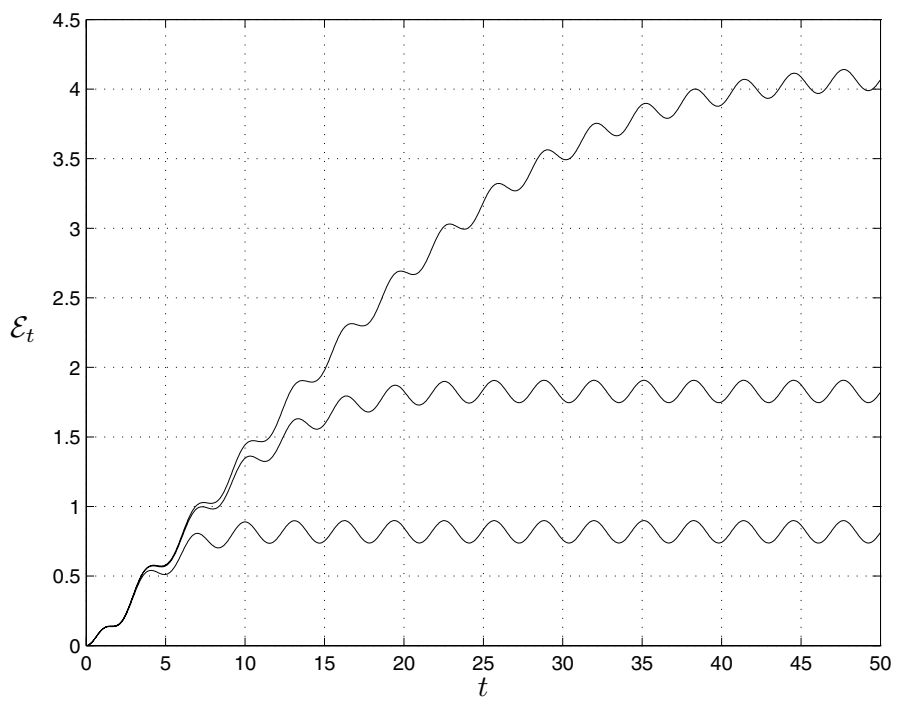

Fig. 3. Temporal growth of the total wave energy for three values of viscosity: from bottom to top $v=10^{-3}, 10^{-4}, 10^{-5}$. Both the time required to reach the permanent regime and the final average energy stored in the system decrease as $v^{-1 / 3}$.

physical effects. We concentrate mainly on the low Fourier harmonics since firstly, these receive the bulk of the energy supplied by the driver; and secondly, long wavelength disturbances are the most difficult to dissipate by visco-resistive processes. When strong dissipation does occur, it is due to the steep cross-field gradients produced by phase mixing. This suggests that only the dominant cross-field derivatives need to be retained. We also make the physically plausible assumption that the driver $V_{0}(y)$ has a global spatial scale much larger than any dissipation length scale. In this case the contribution of the driver to "in-situ" viscous dissipation (see (9)) can also be neglected.
With the above assumptions the modal system reduces to (for notational simplicity we drop the mode number subscript $n$ in the $W$ and $Z$ fields)

$$
\begin{aligned}
\frac{\partial W}{\partial t} & =-n \omega_{\mathrm{A}}(y) Z+v \frac{\partial^{2} W}{\partial y^{2}}-s_{n} \frac{\partial V}{\partial t}, \\
\frac{\partial Z}{\partial t} & =n \omega_{\mathrm{A}}(y) W+\eta \frac{\partial^{2} Z}{\partial y^{2}} .
\end{aligned}
$$

System (14)-(15) is fourth order when both resistivity and viscosity are present and therefore not amenable to convenient analytic description. As far as the permanent regime is concerned, however, our computations show that the system behaves comparably if either resistivity or viscosity is switched off. We therefore specialize to the case of negligible resistivity in the following analysis.

\subsection{General solution in the permanent regime}

In the case $\eta=0$ it is possible to write a formal solution for $W(y, t)$, by taking a Laplace transform with respect to time, in which $t \rightarrow p$ (say). The transformed solution can then be expressed as a convolution product involving the source function $V(y, t)$ and the Green's function $G\left(y, y^{\prime}, p\right)$. Although this procedure can be carried out explicitly in the case of the ideal solution of Sect. 2.4, the inversion to recover $W(y, t)$ depends on a detailed knowledge of the singularities of $G$ and is made difficult because the Green's function is not generally expressible as a named analytic function. Even if it were, it seems unlikely that a closed expression detailing the full transient behaviour of $W(y, t)$ for arbitrary $v$ could be recovered. On the other hand the source function introduces poles at $p= \pm \mathrm{i} \Omega$, which accounts for the permanent regime in which all the field lines oscillate at the same frequency $\Omega$. Restricting attention to this stage we write the driving as $\operatorname{Im}\left(V_{0}(y) \mathrm{e}^{\mathrm{i} \Omega t}\right)$ and take $W=\operatorname{Im}\left(F(y) \mathrm{e}^{\mathrm{i} \Omega t}\right)$. Then $F$ satisfies

$-\mathrm{i} \nu \Omega F^{\prime \prime}+\left(n^{2} \omega_{\mathrm{A}}^{2}(y)-\Omega^{2}\right) F=\Omega^{2} s_{n} V_{0}(y)$, 
where the prime denotes the derivative in $y$. This equation provides our basic tool for exploring the oscillatory regime.

Consider for a moment the $W$ and $Z$ fields expressed in terms of the real and imaginary parts of $F(y)$ ( $R$ and $I$ respectively):

$W(t, y)=R(y) \sin \Omega t+I(y) \cos \Omega t$

$Z(t, y)=\frac{n \omega_{\mathrm{A}}}{\Omega}(I(y) \sin \Omega t-R(y) \cos \Omega t)$.

We see from (18) that energy equipartition is respected only at the resonant line where $n \omega_{\mathrm{A}}=\Omega$. For field lines whose eigenfrequency is higher than $\Omega$ the magnetic energy dominates the kinetic energy. Conversely, for field lines sufficiently close to the neutral line satisfying $y \ll y_{\mathrm{r}}$ the energy is almost entirely kinetic.

Returning to the study of (16), we note that only special forms of $\omega_{\mathrm{A}}$ admit solutions in terms of named functions. Even in the simplest case where $\omega_{\mathrm{A}} \propto y$ the general solution involves parabolic cylinder functions which must be superposed to approximate the actual smooth solution. We find a more direct and informative method is provided by the technique of matched asymptotic expansions (Ruderman et al. 1997; Ruderman 1999).

\subsection{Local solution around the resonance}

It is useful to rewrite (16) in the form

$\mathrm{i} v \Omega F^{\prime \prime}+K(y) F=S(y)$,

with $K(y)=n^{2} \omega_{\mathrm{A}}^{2}(y)-\Omega^{2}$ and $S=\Omega^{2} s_{n} V_{0}$. Note that advective term $K$ vanishes at $y=y_{\mathrm{r}}$, and that $S$ is assumed to vary only over a global scale $(O(1))$. Equation (19) has been already considered by Ruderman et al. (1997), working in cylindrical geometry, in the case where $K(y)$ varies linearily around the resonance $y_{\mathrm{r}}$. We first summarize their approach before discussing the limitations of the linear approximation and generalizing to more complicated forms of $K(y)$.

Figure 4 represents the variation of the function $K$ in $y$ for different driving frequencies $\Omega$, assuming a sinusoidal profile for $v_{\mathrm{A}}$. Two cases will be considered depending on how $\omega_{\mathrm{A}}$ varies around the resonant point. In the first place we assume that $K$ can be linearized according to $K(y)=a\left(y-y_{\mathrm{r}}\right)$ with $a$ of order unity (see top panel). In the next section, we analyze the case where the driving frequency is chosen such that the resonant line corresponds to a maximum of $\omega_{\mathrm{A}}$. Then $K(y)$ is quadratic around $y=y_{\mathrm{r}}$ (see central panel of Fig. 4). The bottom panel of Fig. 4 shows the limits of the linearization. When $\Omega$ is very small, the resonant line is located very close to the neutral sheet and the linearizing $K(y)$ around that point represents a poor approximation to the actual variation. In this case the analytic treatment given below is expected to break down for very low frequencies.

Ruderman et al. (1997) showed that viscous effects are expected to play an important role only in a narrow region of width $\delta$ about the resonant line $y=y_{\mathrm{r}}$. The width of the resonant layer can be obtained by comparing the advective and diffusive terms in (19):

$\delta \equiv\left(\frac{\nu \Omega}{a}\right)^{1 / 3}$.

Outside this region the viscous effect may be ignored and the ideal solution remains valid

$F_{\text {out }}(y)=\frac{S}{K}=\frac{\Omega^{2}}{n^{2} \omega_{\mathrm{A}}^{2}(y)-\Omega^{2}} s_{n} V_{0}(y)$.
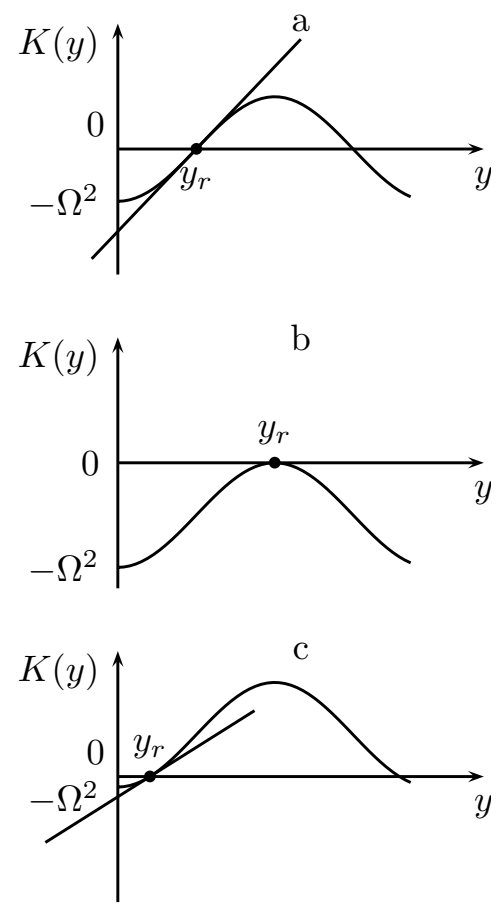

Fig. 4. Sketch of the function $K(y)=n^{2} \omega_{\mathrm{A}}^{2}(y)-\Omega^{2}$ in the case of a sinusoidal profile for various driving frequency $\Omega$. The resonant line corresponds to the point $K=0$. a) General case where the function $K$ can be linearized accurately around the resonance; $\mathbf{b}$ ) case of a quadratic variation around the resonant point; $\mathbf{c}$ ) when $\Omega$ is small, the linearization is no longer a good approximation of $K$ on the diffusion scale $\delta$.

Inside the dissipation region $K=a\left(y-y_{\mathrm{r}}\right)$ and $S \simeq S\left(y_{\mathrm{r}}\right)$ can be considered constant. Then (19) becomes

$\mathrm{i} \nu \Omega F_{\text {in }}^{\prime \prime}+a\left(y-y_{\mathrm{r}}\right) F_{\text {in }}=S\left(y_{\mathrm{r}}\right)$.

Introducing

$z=-\mathrm{i} \frac{y-y_{\mathrm{r}}}{\delta} \quad$ and $\quad \mathcal{S}=\frac{\delta^{2} S\left(y_{\mathrm{r}}\right)}{\nu \Omega}$

and using the definition of $\delta$ we get

$F_{\text {in }}^{\prime \prime}-z F_{\text {in }}=$ i $\mathcal{S}$.

This last Eq. (24) is an inhomogeneous Airy equation whose solution can be written as (Abramowitz \& Stegun 1970):

$F_{\text {in }}=\mathrm{i} \pi \mathcal{S H i}(z)+\alpha \operatorname{Ai}(z)+\beta \operatorname{Bi}(z)$,

where $\alpha$ and $\beta$ are two constants and $\mathrm{Ai}, \mathrm{Bi}$ and $\mathrm{Hi}$ are Airy functions.

The inner solution remains valid as long as the first order expansion of $K$ holds, i.e. when

$$
\begin{gathered}
K^{\prime \prime}\left(y_{\mathrm{r}}\right)\left|y-y_{\mathrm{r}}\right|^{2} \ll 2 K^{\prime}\left(y_{\mathrm{r}}\right)\left|y-y_{\mathrm{r}}\right| \\
\left|y-y_{\mathrm{r}}\right| \ll 2 K^{\prime} / K^{\prime \prime} \ll 1 .
\end{gathered}
$$

The condition is not severe and overlaps the range of validity of the outer solution as long as the resonant line is not too close to the neutral sheet (see Fig. $4 \mathrm{c}$ ) where both $K^{\prime}$ and $K^{\prime \prime}$ vanish. There is thus a common interval $\delta \ll y-y_{\mathrm{r}} \ll 1$ where the two expressions (21) and (25) represent the same solution and must be matched asymptotically as $v \rightarrow 0$. This matching implies that the coefficients $\alpha$ and $\beta$ should be identically zero. Note that Ruderman et al. (1997) writes the solution (25) in the form of its integral representation but both results are in exact agreement. 

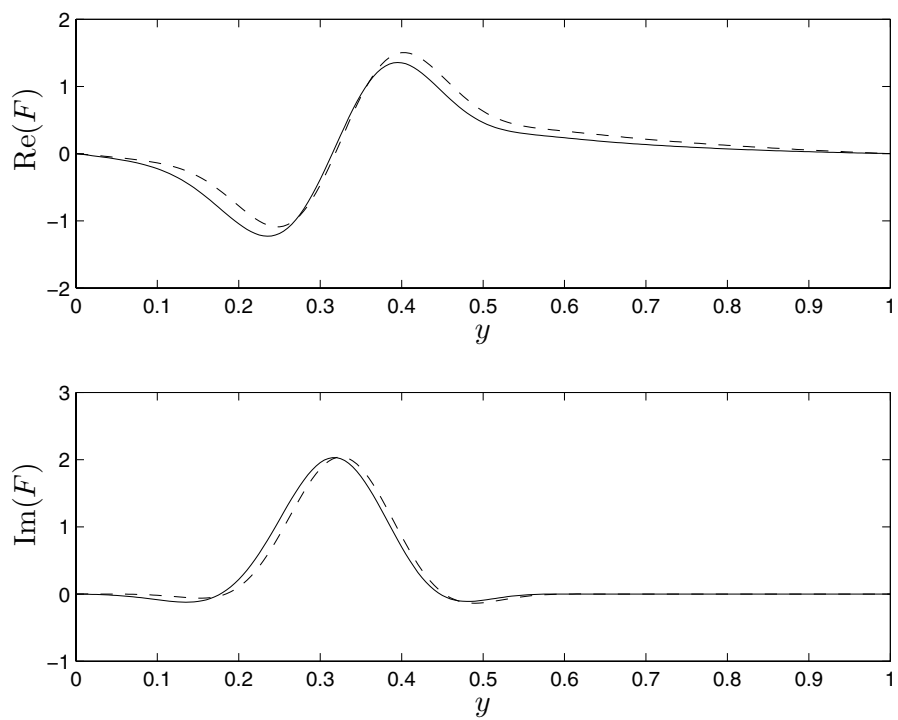

Fig. 5. Real and imaginary parts of function $F$ computed directly from Eq. (19) (solid line) and compared with the analytical expression (26). Following values have been chosen: $v=10^{-3}, \Omega=1$, a linear profile for the Alfvén speed and $V_{0}(y)=\sin \pi y$. The agreement between the two curves over the whole spatial domain confirms that the asymptotic matching approximation provides an excellent description of the actual perturbation.

We conclude that an inner region of width $\delta$ centred on $y_{\mathrm{r}}$ can be defined where the perturbation field takes the form

$F_{\text {in }}(y)=\frac{\mathrm{i} \pi}{v} s_{n} \Omega V_{0}\left(y_{\mathrm{r}}\right) \delta^{2} \mathrm{Hi}\left(-\mathrm{i} \frac{y-y_{\mathrm{r}}}{\delta}\right)$.

For sufficiently small viscosity, this expression matches the ideal solution in the outer region $\left|y-y_{\mathrm{r}}\right|>\delta$. In fact Fig. 5 indicates that this approximation holds good over the entire interval, provided only that $V_{0}\left(y_{\mathrm{r}}\right)$ is replaced by $V_{0}(y)$. This comparison, which is based on the Alfvén profile $\omega_{\mathrm{A}}=\pi y$ with $v=10^{-3}, \Omega=1$, and $V_{0}(y)=\sin \pi y$, shows that the Airy function can represent the global solution (19) reasonably well even when $v$ is not especially small. Of course, the agreement improves progressively as $v \rightarrow 0$.

Finally, we mention the viscous scaling laws that derive naturally from the analysis. We see that the width of the resonance line where most of the dissipation occurs scales as $v^{1 / 3}$. This scaling leads to an energy dissipation rate which is independent of $v$ (see Sect. 3.5). However, this favourable result should be balanced against the amplitude of $F$ - and thus the axial wave energy - which, according to (24), builds up as $v^{-1 / 3}$. The interpretation of these scalings is discussed further in Sect. 3.5 below.

\subsection{Particular case of an extremum in the Alfvén velocity profile}

We now consider the case where the Alfvén velocity profile is maximum at the resonant line $y_{\mathrm{r}}$, so that the advective term can be approximated by a quadratic expression $K(y)=b\left(y-y_{\mathrm{r}}\right)^{2}$ with $b$ a constant of order unit. The width of the diffusive zone is again determined by comparing the advective and diffusive terms:

$\delta \equiv\left(\frac{\nu \Omega}{b}\right)^{1 / 4}$.
This scaling suggests that the region of resonance is broadened slightly when the line is centred around a maximum in the Alfvén velocity.

In the inner region of width $\delta$ Eq. (19) reduces to

$\mathrm{i} \nu \Omega F_{\text {in }}^{\prime \prime}+b\left(y-y_{\mathrm{r}}\right)^{2} F_{\text {in }}=S\left(y_{\mathrm{r}}\right)$.

With

$z=\frac{y-y_{\mathrm{r}}}{\delta} \quad$ and $\quad \mathcal{S}=\frac{\delta^{2} S\left(y_{\mathrm{r}}\right)}{\nu \Omega}$

we find that the analogue of Eq. (24) is

$F_{\text {in }}^{\prime \prime}-\mathrm{i} z^{2} F_{\text {in }}=-\mathrm{i} S$.

In this case the solution can be expressed in terms of Bessel functions of order 1/4 (see Abramowitz \& Stegun 1970):

$F_{\text {in }}=-\mathrm{i} \pi \mathcal{S} \int_{0}^{z}\left[J_{1 / 4}(u) Y_{1 / 4}(z)-J_{1 / 4}(z) Y_{1 / 4}(u)\right] \mathrm{d} u$.

This expression may be compared with solution (26) based on the linear approximation. Since the Airy function Hi can be written as an integral over Bessel functions of order $1 / 3$, we see that the passage from a linear to a quadratic Alfvén profile is reflected in some function - say $H$ - which can be expressed in terms of fractional order Bessel functions. In particular, the generic form

$F_{\text {in }}(y)=\mathrm{i} \pi s_{n} \frac{\Omega \delta^{2}}{v} V_{0}\left(y_{\mathrm{r}}\right) H\left(\frac{y-y_{\mathrm{r}}}{\delta}\right)$

accounts for both (26) and (31). The scaling with viscosity is contained in the dependence of $\delta$ on $v$. In the quadratic case we have $\delta \sim v^{1 / 4}$ and so the amplitude of perturbation fields scales as $\delta^{2} / v \sim v^{-1 / 2}$.

\subsection{Dissipation rate around the resonance}

We now explore how the energy dissipation rate scales with viscosity. Since the dissipation is most efficient near the resonant line, we can simplify the calculation by taking

$\mathcal{W}=-\int_{y_{\mathrm{r}}-\delta}^{y_{\mathrm{r}}+\delta} v\left(\frac{\partial W_{n}}{\partial y}\right)^{2} \mathrm{~d} y$.

Using expressions (17), (32) and the change of variable $u=(y-$ $\left.y_{\mathrm{r}}\right) / \delta$, this becomes

$\mathcal{W}=-\int_{y_{\mathrm{r}}-\delta}^{y_{\mathrm{r}}+\delta} v\left|F_{\mathrm{in}}^{\prime}\right|^{2} \mathrm{~d} y=-\pi^{2} s_{n}^{2} \frac{\Omega^{2} \delta^{3}}{v} V_{0}^{2} \int_{-1}^{+1}\left|H^{\prime}\right|^{2} \mathrm{~d} u$.

The remaining integral is simply a number which cannot affect the scaling with viscosity.

For the linear Alfvén profile of Sect. 3.3, we recall that inner region scales as $\delta \sim v^{1 / 3}$. In this case (34) confirms that the dissipation rate scales independently of the viscosity. Physically, the system behaves like a high quality resonator: viscous effects may influence the initial development of the resonance layer, but once the layer is established, the dissipation rate scales as $v^{0}$.

Figure 6 displays the dissipation rate $W$ as a function of time for three values of viscosity $\left(v=10^{-3}, v=10^{-4}, v=10^{-5}\right)$. It does indeed take longer to reach the permanent regime for small $v$, but in all cases the same dissipation rate is achieved. This result could be very important in the context of coronal heating. 


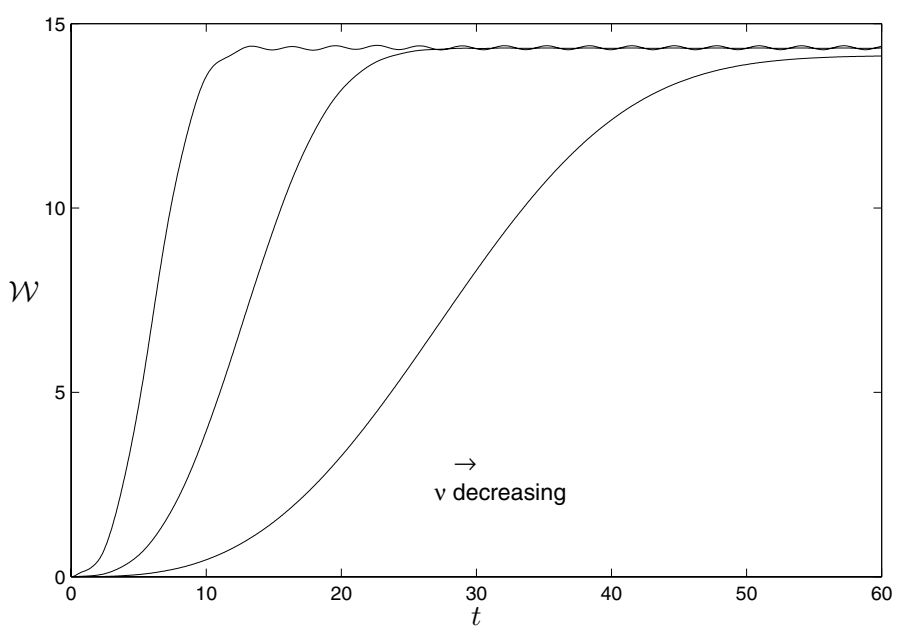

Fig. 6. Temporal growth of the dissipation rate $\mathcal{W}$ for three values of viscosity: from left to right $v=10^{-3}, 10^{-4}, 10^{-5}$. Although it takes longer to reach the permanent state in a low viscous plasma, the final dissipation rate does not depend on the viscosity.

However, this result should be tempered by the fact that the wave energy $\mathcal{E} \simeq W^{2} \delta$ ) scales as $v^{-1 / 3}$. For very small viscosities, the amount of energy stored in the oscillator may become tremendous and non physical, unless some feedback mechanism moderates the driver. We will show in paragraph 3.6, however, that this drawback can be eluded by judicious choice of the driving frequency.

Before this discussion, we would like to point out a rather strange result of our analysis. When the resonance line corresponds to a maximum in the Alfvén velocity profile - we use here a sinusoidal function of the form $v_{\mathrm{A}}=\sin \pi y$ which is maximum at $y=0.5-$, the diffusive region broadens $\delta \sim v^{1 / 4}$ and the dissipation rate (34) scales as $v^{-1 / 4}$. It means that the less viscous a plasma is, the more energy it dissipates during one oscillation period. This surprising behaviour is yet confirmed by Fig. 7 which displays as a function of $v$ the maximum dissipation rate obtained for $\Omega=\pi\left(v_{\mathrm{A}}\right)_{\max } / L=\pi$ if $L=1$.

Once more, to interpret these results correctly we should bear in mind two points: first, that the permanent state takes longer to achieve with a quadratic profile because the relatively weak variation of the Alfvén velocity does not enable strong phase mixing around the maximum; second, that the global wave energy is divergent, $\mathcal{E} \propto W^{2} \delta \sim v^{-1 / 2}$ as $v \rightarrow 0$. Nonetheless, in this particular situation where the driving frequency corresponds to a local maximum in the Alfvén profile, the driver supplies globally more energy to the oscillator than it does in other circumstances. Consequently, these regions of maximum Alfvén velocity could constitute favorable sites for wave energy deposition.

\subsection{Interpretation using the quality factor}

According to the previous results, the rapid dissipation of wave energy in the sheet relies on an "external driver" whose properties are decoupled from the wave dissipation dynamics of the current sheet. To establish the resonant regime, the driver must feed energy into the sheet and both the setting up time of the resonance line, and the saturation of the global wave energy, depend on the level of the dissipation. In particular, the super fast dissipation obtained for the quadratic Alfvén profile $\mathcal{W} \sim v^{-1 / 4}$, is achieved at the expense of wave energies that scale as $v^{-1 / 2}$. A similar conclusion holds for the resonance line associated with the linear Alfvén profile, despite the relatively modest $\left(v^{-1 / 3}\right)$

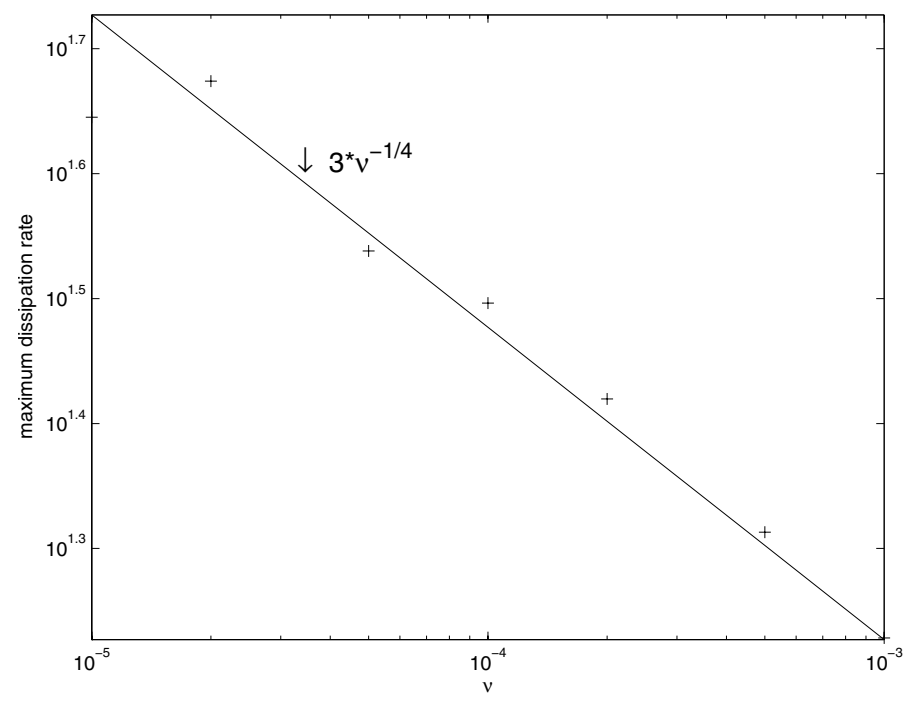

Fig. 7. Variation of the dissipation rate with viscosity in the case of a parabolic profile around the resonance. The $\log \log$ fit shows that $\mathcal{W}$ scales as $v^{-1 / 4}$.

build up in global wave energy. Since this behaviour is not sustainable in the limit $v \rightarrow 0$ we might expect some feedback mechanism to moderate the driver. Specifically, if footpoint motions are assumed to provide the external energy source, then such motions will be influenced if wave energy densities in the resonance line become comparable to energy densities in the "external" regions governing the driver. On the other hand, our analytic treatment does not explore the whole range of frequencies. For example, low driving frequencies $\Omega$ excite resonant lines that lie very close to the neutral sheet $y=0$. In this situation, the linearization of the function $K$ (see Sect. 3.3) represents a very crude approximation of the parabola as displayed in Fig. 4. Bearing in mind that the scaling laws derived in 3.3 are unlikely to be reliable in this case, it is wise to explore the limit of low frequency driving using numerical methods.

Our aim is to quantify the energy dissipation versus the amount of global wave energy as $\Omega$ becomes small. For that matter, we can introduce the quality factor $Q$ as the ratio between the average total energy stored in the oscillator and the dissipation rate over one period,

$Q=\frac{\mathcal{E}}{W \frac{2 \pi}{\Omega}}$.

In some resonant systems, such as a laser cavity or an electric circuit, energy losses are considered a nuisance and the quality factor should be chosen as high as possible. In this case it may be possible to sharpen the resonance by tuning to a narrow band of favourable frequencies. Here, on the contrary, we are mainly interested in the effectiveness of the energy dissipation, so our aim is to keep $Q$ as low as possible (soft resonance).

In top panel of Fig. 8 we show the quality factor $Q$ as a function of the driving frequency $\Omega$ for five values of $v$ ranging from $10^{-7}$ to $10^{-3}$. For this computation, a linear Alfvén profile is used. All curves display a minimum for a frequency around $\Omega_{\text {min }} \sim 0.1$, the precise position depending on the viscosity. This minimum corresponds to a resonant line lying very close to the neutral line. Although, for moderate (fixed) $\Omega \gg \Omega_{\min }$, the quality factor reflects the expected $v^{-1 / 3}$ scaling, the analytic theory begins to break down for $\Omega \rightarrow \Omega_{\min }$. Note, in particular, that when the viscosity is large, the quality factor is relatively insensitive to the driving frequency. In this case $Q \simeq 1$ is achieved 

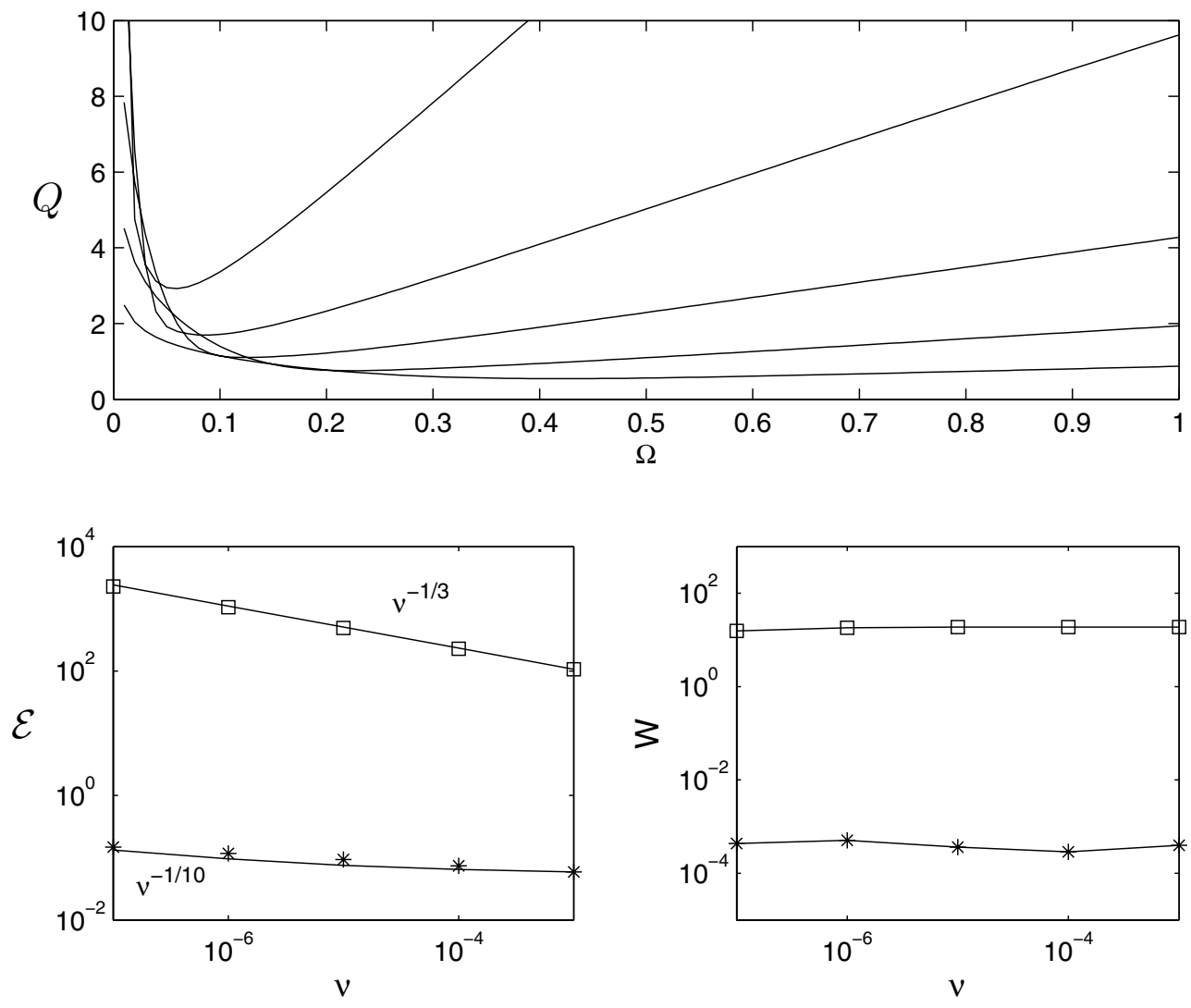

Fig. 8. Top panel: plot of the quality factor (35) versus the driving frequency $\Omega$ for five values of viscosity: from top to bottom $v=10^{-7}, 10^{-6}, 10^{-5}$, $10^{-4}, 10^{-3}$. A linear Alfvén profile is used here and the source term has a sinusoidal dependence dependance in $y$ : $V_{0}=\sin \pi y$. A minimum is clearly visible for each curves and sharpens as $v$ decreases. Near this minimum the dissipation of energy is optimal. Bottom left panel: global wave energy stored in the current sheet as a function of $v$ for $\Omega=0.9$ (squares) and $\Omega=0.06$ (stars). Wave energy scales according to a power law in both cases, but the exponent becomes very small at low frequency. Bottom right panel: dissipation rate as a function of $v$ with the same pattern as for the energy. The plot confirms that the dissipation rate remains independent of the viscosity whatever the frequency is. for a broad range of frequencies and the oscillator can dissipate, over one period, as much energy as it stores. As $v$ decreases however, the resonance becomes more sensitive to the driver and $Q$ begins to rise. Yet even with viscosity as weak as $v=10^{-7}$, a range of frequencies can be found where $Q$ remains quite small $Q<3$.

We conclude that, as far as the minimum quality factor is concerned, the problem may be quite insensitive to the viscosity. The implication is that, by tuning the driver, we can achieve a response which is favourable, both in terms of the stored wave energy and the dissipation rate. This interpretation is confirmed in the bottom panels of Fig. 8. The energy stored remains moderate in a very low viscous plasma if the oscillator is driven at low frequency $\Omega \sim 0.1$ which corresponds, according to the coronal parameters of Sect. 2.1, to a driving period of around $10 \mathrm{~s}$, while the dissipation rate $\mathcal{W}$ is still independent of the viscosity. Of course, to be of practical significance for heating the corona, we must suppose that the power spectrum of the driver is capable of supplying significant energy in a band around this frequency.

\section{Discussion and conclusion}

In this paper we have considered the viscous and resistive energy dissipation of externally-driven, shear Alvén waves in a line-tied magnetic channel. An important aspect of the geometry is the inhomogeneity of the background magnetic field. Specifically, the presence of a neutral line at $y=0$ allows perturbations to develop strong, cross-field gradients by phase mixing. It follows that waves generated by quasi-periodic motions of the footpoints can be damped very effectively, despite the smallness of the dissipation coefficients $(v$ and $\eta$ ). The wave damping can be "fast" - that is, independent of the viscous and resistive dissipation coefficients.
We have concentrated mainly on the permanent "resonance" regime established by cyclic, shearing motions of the footpoints. The system can resonate when it contains field lines that oscillate at a frequency $\Omega$ that matches the external driver. In our model $\Omega \leq 1$, corresponding to Alfvénic periods of a several seconds or greater (in the low corona, say), defines the resonance band of the fundamental global oscillation. Once the system is driven into its permanent regime, the dissipation rate becomes insensitive to the level of the viscous and resistive damping. The actual scaling of the rate depends on the form of the Alfvénic profile $v_{\mathrm{A}}(y)$ and for quadratic (as opposed to linear) profiles the dissipation rate can actually increase with $v$ (assuming viscosity is the dominant damping mechanism). But in order to set up the conditions for resonance, the magnetic channel must be able to absorb energy continually from the external driver. The level at which the energy density in the channel saturates is found to depend inversely on the magnitude of the damping coefficient. It follows that, to avoid unrealistic energies at low damping levels, some feedback must be present between the driver and resonance region. In fact the build-up of energy may not be too serious in practice, given the relatively modest energy accumulation $\left(\mathcal{E} \sim v^{-1 / 3}\right.$ for $\left.v_{\mathrm{A}}(y) \sim y\right)$ and the fact that the dimensionless viscosity can be as large as $10^{-5}$ for plasmas in the solar atmosphere.

We have also pointed out, however, that the rise in global wave energy as $v \rightarrow 0$ can be mitigated by tuning the system to a sufficiently low driving frequency $\Omega<0.1$, corresponding to driving timescales exceeding $10 \mathrm{~s}$ for the low corona. The response of the system - in terms of both the stored wave energy and the dissipation rate - then becomes largely independent of damping. In this case the resonance region may be capable of quite rapid energy dissipation, perhaps approaching flare-like 
scales of a few hundred seconds, without accumulating large energies in the resonance channel.

In practise of course, the spectrum of driving frequencies is thought to be determined by the buffeting of magnetic footpoints in the low solar atmosphere. For the present mechanism to be effective there must be sufficient power in the driver, particularly at low frequencies. The Fourier spectrum associated with footpoint disturbances is largely unknown, but it seems likely that low frequency modes will supply the bulk of the power (Milano \& Gomez 1997). What can be said is that the present study supports the view that energy residing mainly in the low frequency modes may be absorbed within a resonating magnetic channel and rapidly dissipated by visco-resistive damping. The model therefore reinforces the view that the energy supplied by footpoint motions in the low chromosphere may act as a source for coronal heating.

Acknowledgements. This work was supported by the Marsden Fund (02-UOW050 MIS).

\section{References}

Abramowitz, M., \& Stegun, I. A. 1970, Handbook of Mathematical Functions (National Bureau of Standards)

Athay, R. G., \& White, O. R. 1978, ApJ, 226, 1135

Berghmans, D., De Bruyne, P., \& Goossens, M. 1996, ApJ, 472, 398

Braginskii, S. I. 1965, Rev. Plasma Phys., 1, 205

Craig, I. J. D., \& Fruit, G. 2005, A\&A, 440, 357

Fruit, G., \& Craig, I. J. D. 2006, A\&A, 448, 753

Fruit, G., Louarn, P., Tur, A., \& LeQuéau, D. 2002, 107

Heyvaerts, J., \& Priest, E. R. 1983, A\&A, 117, 220

Hollweg, J. V. 1986, ApJ, 306, 730

Hollweg, J. V. 1987, ApJ, 320, 875

Hood, A. W., Gonzalez-Delgado, D., \& Ireland, J. 1997, A\&A, 324, 11

Ionson, J. A. 1978, ApJ, 226, 650

Mikic, Z., Schnack, D., \& van Hoven, G. 1989, ApJ, 338, 1148

Milano, L., \& Gomez, D. 1997, ApJ, 490, 442

Priest, E. R., \& Forbes, T. 2000, Magnetic Reconnection (Cambridge U Press)

Ruderman, M. S. 1999, ApJ, 521, 851

Ruderman, M. S., Berghmans, D., Goossens, M., \& Poedts, S. 1997, A\&A, 320, 305

Spitzer, L. 1962, Physics of Fully Ionized Gases (John Wiley \& Sons)

Tirry, W. J., Berghmans, D., \& Goossens, M. 1997, A\&A, 322, 329 